\title{
Radiologic criteria of retropharyngeal lymph node metastasis in maxillary sinus cancer
}

\author{
Yasuo Kosugi ${ }^{1 *} \mathbb{D}$, Michimasa Suzuki ${ }^{3}$, Mitsuhisa Fujimaki², Shinichi Ohba², Fumihiko Matsumoto², \\ Yoichi Muramoto ${ }^{1}$, Terufumi Kawamoto ${ }^{1}$, Masaki Oshima ${ }^{1}$, Naoto Shikama ${ }^{1}$ and Keisuke Sasai ${ }^{1}$
}

\begin{abstract}
Objective: To determine the most appropriate radiologic criteria of metastatic retropharyngeal lymph nodes (RLNs) in patients with maxillary sinus cancer (MSC).

Materials and methods: We retrospectively evaluated 16 consecutive patients who underwent magnetic resonance imaging (MRI) before and after the treatment of locally advanced squamous cell carcinoma of the maxillary sinus. The minimal and maximal diameters of all RLNS were recorded. RLNs were classified as metastatic on the basis of the MRI follow-up ( $f / u$ ). RLNs were considered non-metastatic if stable disease continued until the final MRI f/u and metastatic in cases with different evaluations (complete response, partial response, progressive disease) determined using Response Evaluation Criteria in Solid Tumours (RECIST) ver. 1.1. The receiver operating characteristic curve (ROC) and area under the curve (AUC) were used to assess the accuracy of various criteria in the diagnosis of metastatic RLNs.
\end{abstract}

Results: Of the 34 RLNs in 16 cases observed on pretreatment MRI, 7 were classified as metastatic RLNs and 27 as non-metastatic RLNs. Using the radiologic criteria, metastatic RLNs tended to be diagnosed more accurately with the minimal axial diameter than with the maximal axial diameter ( $A \cup C ; 0.97 \mathrm{vs} .0 .73, p=0.06$ ). The most accurate size criterion of metastatic RLNs was a minimal axial diameter of $5 \mathrm{~mm}$ or larger, with an accuracy of $94.1 \%$ (32 of 34).

Conclusions: The most appropriate radiologic criterion of metastatic RLNs in MSC is a minimal axial diameter of $5 \mathrm{~mm}$ or longer.

Keywords: Maxillary sinus cancer, Retropharyngeal lymph node metastasis, Radiologic criteria, Magnetic resonance image

\section{Introduction}

Maxillary sinus cancer (MSC) is a relatively rare disease, with an incidence of 1 per 100000 person-years, and accounts for $3 \%$ of all head and neck cancers. Most MSC cases are squamous cell carcinoma (SCC), representing $60 \%$ to $90 \%$ of total cases [1]. Tobacco use is a significant risk factor. The prognosis of MSC with lymph node (LN) metastases is significantly poor. Five-year overall survival (OS) of patients with SCC of the paranasal sinuses is

*Correspondence: ykosugi@juntendo.ac.jp

1 Department of Radiation Oncology, Juntendo University, 2-1-1 Hongo, Bunkyo-ku, Tokyo 113-8421, Japan

Full list of author information is available at the end of the article approximately 50\%, and 30\% among those with localized and regional disease [2].

Surgery and (chemo)radiation therapy are recommended as the standard treatments for MCS [2]. The combination of radiation therapy and intra-arterial (IA) chemotherapy is a promising treatment for patients who had unresectableMSC or refused surgery because of its high control rate and high OS [3, 4]. This is a popular treatment in Japan, and although it is not a standard method, we have observed good results in patients treated with locally advanced MSC using this combination [5]. In this setting, we have performed elective nodal irradiation (ENI) only for patients with regional LN metastasis $(\mathrm{cN}+)$. From the results of a meta-analysis, 
the guidelines of the National Comprehensive Cancer Network (NCCN) recommended ENI for patients with advanced MSC [6], but ENI is controversial because of reports of increased late adverse events comprising dysphagia and trismus [7]. We reported that ENI increased the radiation dose in the pharyngeal contractile muscles and increased late aspiration pneumonia [8]. The irradiation field setting of the retropharyngeal lymph node (RLN) region is important because it affects the radiation dose of the pharyngeal contractile muscle and may cause severe acute mucositis and late dysphagia in ENI [9-12]. Recently, intensity-modulated radiation therapy (IMRT) for MSC has been reported to be useful in reducing adverse events [2]. Similarly, IMRT highlights the risk of marginal recurrence, and a more accurate irradiation field setting is required [13]. Because of the high frequency of metastatic RLNs detected by magnetic resonance imaging (MRI) in recent years [14], the European Society for Radiotherapy \& Oncology (ESTRO) guidelines recommended including the RLN area as a prophylactic region for radiation therapy in MSC [15]. However, radiologic criteria for metastatic RLNs in MSC have not been established.

Therefore, in this study, we determined metastatic RLNs on the basis of the therapeutic response of RLNs using MRI images taken before and after treatment and investigated the appropriate radiologic criteria for metastatic RLNs in MSC.

\section{Patients and methods}

\section{Study design and data collection}

We retrospectively analyzed the clinical data of 16 consecutive patients who underwent MRI before and after treatment among 55 patients with locally advanced SCC of the maxillary sinus who were treated with definitive external beam radiotherapy and IA chemotherapy from April 2009 to August 2017 at our institution. All patients had unresectable MSC or refused surgery. The ethics committee of our hospital approved the study protocol (approval number: 19-173), and the study was conducted in accordance with the principles of the Declaration of Helsinki. Furthermore, staging was performed on the basis of findings from physical examinations, computed tomography (CT), MRI, and/or positron emission tomography-CT (PET-CT), in accordance with the Union for International Cancer Control (UICC, $7^{\text {th }}$ edition) [16].

\section{Treatment}

Details of definitive external radiotherapy and superselective IA chemotherapy were given in our previous report [7]. In $\mathrm{cN}+$ cases, the primary sites and metastatic LNs were irradiated with 60-70 Gy (median, 60 Gy, to iso-center by three-dimensional conformal radiation therapy (3DCRT), prescription dose $>50 \%$ of the target volume by IMRT) in 30-35 fractions over 6-7 weeks, and the prophylactic regional area (ENI) was irradiated with 46 Gy in 23 fractions by 3DCRT or 50 Gy in 25 fractions by IMRT (median, 46 Gy). ENI was not performed in clinical node-negative cases. Ipsilateral levels I, II, and III were set for regional irradiation, and contralateral levels II and III were also added in cases of bilateral LN metastases at presentation. Bilateral RLN regions were added for regional irradiation in IMRT cases.

\section{Imaging protocol}

Of 16 patients, 11 underwent MR imaging with a 3-Tesla system (Achieva or Ingenia; Royal Philips, Amsterdam, Netherlands) employing a three-dimensional gradientecho technique. The entire RLN region was examined with a head and neck-combined coil. After intravenous injection of gadolinium contrast agent (Magnescope; Guerbet Japan, Tokyo, Japan) at a dose of $0.2 \mathrm{mmol}$ per kilogram of body weight, T1-weighted fat-suppressed axial, coronal, and sagittal sequences were performed sequentially. The most frequently used section thicknesses and intersection gaps were $2 \mathrm{~mm}$ and $0.9 \mathrm{~mm}$, respectively, for the axial plane.

\section{Image assessment}

All MRI images were evaluated by a radiation oncologist and a radiologist (Y.K. with 10 years of experience in MSC MRI imaging and radiation therapy, and M.S. with 19 years of experience in MSC MRI imaging and super-selective IA treatment). If there was a discrepancy between the findings, they were discussed, and a consensus result was recorded. Details of cranial level and ipsilateral or contralateral side were recorded for all visible RLNs at pre-treatment MRI. The minimal and maximal axial diameters of each RLN were measured. The minimal diameter was defined as the widest diameter of the $\mathrm{LN}$ in the axial plane that was perpendicular to the maximal axial diameter. In addition, findings of central necrosis (marginal ring-shaped contrast enhancement) of RLNs were also recorded as indicative of metastatic RLNs.

\section{Therapeutic response detected by MRI follow-up}

The therapeutic response was first investigated by MRI in all patients within 8 to 12 weeks after the completion of radiation therapy. Then, MRI follow up ( $\mathrm{f} / \mathrm{u}$ ) was undertaken within 3 to 12 months. Therapeutic response was classified into complete response (CR), partial response (PR), stable disease (SD), and progressive disease (PD) with reference to the Response Evaluation Criteria in Solid Tumors (RECIST) ver. 1.1, as follows: disappearance, $\mathrm{CR}$; reduction in maximal axial 
diameter by $30 \%$ or more, PR; maximal axial diameter increased by $20 \%$ or more PD; no change, SD [17]. An RLN was considered as metastatic if it was classified as $\mathrm{CR}, \mathrm{PR}$, or PD at any MRI $\mathrm{f} / \mathrm{u}$, and was not considered a metastatic LN if SD continued until the final MRI $\mathrm{f} / \mathrm{u}$ without local recurrence. For RLNs that were changed at the initial MRI after treatment but were evaluated as $\mathrm{SD}$, when the reduction or increase continued in the subsequent additional MRI $f / u$, the pretreatment MRI and the most changed MRI image were compared.

\section{Statistical analysis}

Receiver operating characteristic (ROC) curve and the area under the curve (AUC) were used to determine the most appropriate radiologic criterion of metastatic RLNs in MSC. The sensitivity, specificity, and accuracy were calculated using the standard definitions. The DeLong test was performed to compare the AUC of ROC curves. The most appropriate radiologic criterion was determined using the Youden's index in conjunction with the ROC curve. The Mann-Whitney $U$ test was used to compare the diameters of the metastatic or non-metastatic RLNs. All statistical analyses were assessed at a significance level of 0.05 using JMP 12 software (SAS Institute; Minato-ku, Tokyo, Japan).

\section{Results}

\section{Patients' clinicopathological characteristics}

Table 1 shows patients' characteristics. Pretreatment MRI revealed 34 RLNs in 16 patients. Table 2 shows the details of the anatomical location of these RLNs. All of them were lateral RLNs and there were no medial RLNs.

All patients had completed radiation therapy, and the total number of MRI f/u ranged from 1 to 7 (median, 2 ) over a median observation period of 38 months. The median intersection gap of the MRI axial image was $0.9 \mathrm{~mm}$ (range, 0.7-5.0). No cases had a local recurrence by the time of the final MRI f/u. Therapeutic response detected by the initial MRI after treatment was distributed as follows: CR, two LNs; PR, five LNs; and SD, 27 LNs. Subsequent MRI f/u showed no cases in which the evaluation changed from $\mathrm{SD}$ to $\mathrm{PR}$ or PD. On the basis of the therapeutic response detected by MRI $f / u$, seven RLNs were classified as metastatic lesions and 27 as non-metastatic lesions. The minimal axial diameters of the positive RLNs were greater than those of the negative RLNs (Table 3). Three RLNs showed central necrosis, all of which were positive for metastasis by image f/u (Fig. 1).
Table.1 Patients' characteristics

\begin{tabular}{ll}
\hline Characteristics & $\begin{array}{l}\text { Number } \\
\text { of patients } \\
(\%)\end{array}$ \\
\hline Age & $67(48-81)$ \\
Median (range) & \\
Gender & $13(81)$ \\
Male & $3(19)$ \\
Female & \\
Tstage & $12(75)$ \\
T4a & $4(25)$ \\
T4b & \\
N stage & $6(38)$ \\
N0 & $1(6)$ \\
N1 & $9(56)$ \\
N2b & \\
Pretreatment diagnostic imaging & $16(100)$ \\
MRI & $9(56)$ \\
PET-CT & \\
Radiation thechnique & $9(56)$ \\
3DCRT & $7(44)$ \\
IMRT &
\end{tabular}

\section{Radiologic criteria of metastatic retropharyngeal lymph nodes}

Figure 2 shows the ROC curves of the maximal and the minimal axial diameters. Metastatic RLNs tended to be diagnosed more accurately with the minimal axial diameter than with the maximal axial diameter (AUC $0.97 \mathrm{vs.}$ $0.73, p=0.06$ ). Table 4 shows the sensitivity, specificity, and accuracy for several cut-off values per $\mathrm{mm}$ of the different size criteria. The most accurate radiologic criterion was a minimal axial diameter of $5 \mathrm{~mm}$ or larger. The sensitivity, specificity, and accuracy of this criterion were $85.7 \%, 96.3 \%$, and $94.1 \%$, respectively. In two RLNs, there was a discrepancy between the judgment of metastatic RLNs using this radiologic criterion and that based on the evaluation by MRI $f / u$. That is, the minimal axial diameter was $4.4 \mathrm{~mm}$ before treatment, but the therapeutic effect was CR. Conversely, the minimal axial diameter was $5.9 \mathrm{~mm}$ before treatment, but the therapeutic effect was SD.

\section{Discussion}

There were few reports of RLNs in MSC before 2000 [1], but in recent years there have been reports of a high frequency of RLNs detected by MRI [14]. The inconsistent frequency of metastatic RLNs may be due to changes in diagnostic modalities and the diagnostic criteria [8]. In a previous study, a maximal axial diameter of $10 \mathrm{~mm}$ or 
Table.2 Location of RLNs on pre-treatment MRI

\begin{tabular}{lllll}
\hline Location on MRI & \multicolumn{2}{l}{ No. of RLNs at pretreatment MRI } & \multicolumn{2}{l}{ No. of positive for metastatic RLNs } \\
\cline { 2 - 4 } & Ipsilateral & Contralateral & Ipsilateral & 3 \\
\hline Occipital bone & 3 & 1 & 4 \\
Body of C1 and C1/2 disk & 9 & 6 & - & - \\
Body of C2 and C2/3 disk & 6 & 8 & - \\
Body of C3 & - & 1 & - \\
\hline
\end{tabular}

Table.3 Axial diameter of metastatic or non-metastatic RLNs

\begin{tabular}{lll}
\hline RLNs status & Minimal axial diameter & $\begin{array}{l}\text { Maximal } \\
\text { axial } \\
\text { daiameter }\end{array}$ \\
\hline Positive & $5.9 \pm 0.5$ & $8.8 \pm 1.1$ \\
Negative & $3.4 \pm 0.1$ & $6.5 \pm 0.3$ \\
p value & 0.0001 & 0.06 \\
\hline
\end{tabular}

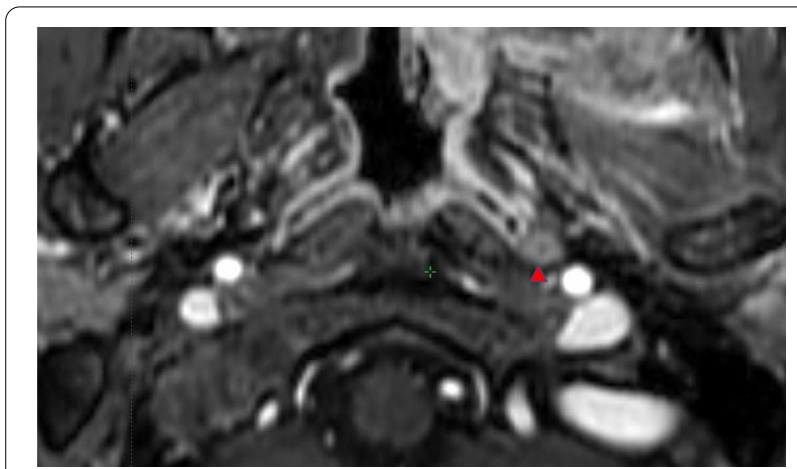

Fig. 1 Metastatic RLN with findings of central necrosis. Arrowheads indicate RLNs with central necrosis

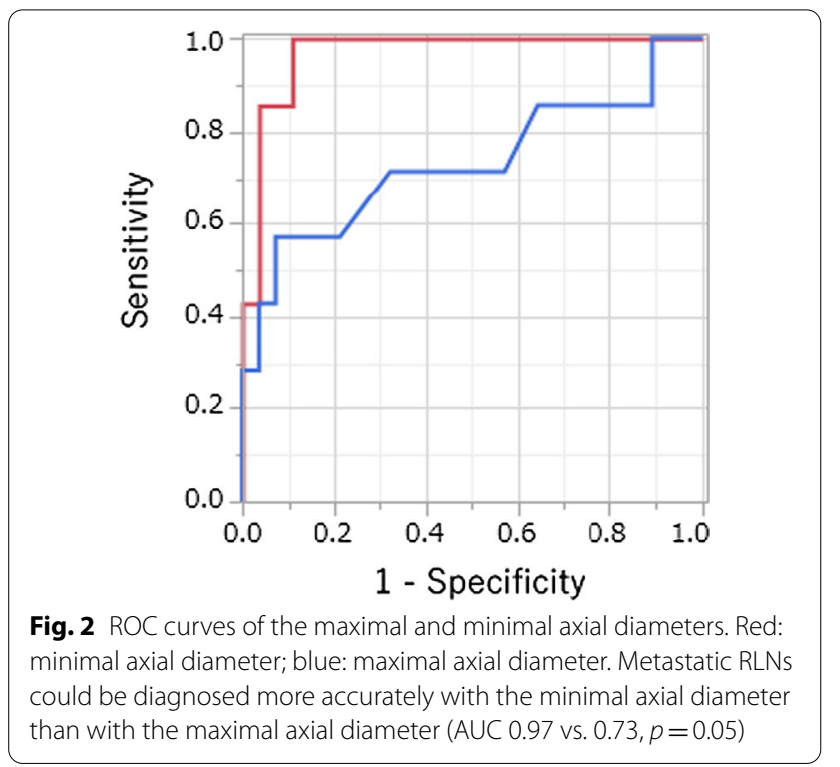

Table.4 Sensitivity, specificity, and accuracy of several cut-offs per $\mathrm{mm}$ of different size criteria

\begin{tabular}{llll}
\hline Diameter $(\mathbf{m m})$ & $\begin{array}{l}\text { Sensitivity(\%) } \\
(\mathbf{n}=\mathbf{7})\end{array}$ & $\begin{array}{l}\text { Specificity } \\
(\mathbf{n}=\mathbf{2 8})\end{array}$ & $\begin{array}{l}\text { Accuracy } \\
(\mathbf{n}=\mathbf{3 5})\end{array}$ \\
\hline \multicolumn{2}{l}{ Minimal axial diameter } & & \\
$\geq 3 \mathrm{~mm}$ & 100 & 22.2 & 38.2 \\
$\geq 4 \mathrm{~mm}$ & 100 & 81.5 & 85.3 \\
$\geq 5 \mathrm{~mm}$ & 85.7 & 96.3 & 94.1 \\
$\geq 6 \mathrm{~mm}$ & 42.9 & 100 & 88.2 \\
$\geq 7 \mathrm{~mm}$ & 14.3 & 100 & 82.3 \\
Maxmal axial daiameter & & \\
$\geq 6 \mathrm{~mm}$ & 71.4 & 44.4 & 50 \\
$\geq 7 \mathrm{~mm}$ & 71.4 & 63 & 64.7 \\
$\geq 8 \mathrm{~mm}$ & 57.1 & 81.5 & 76.5 \\
$\geq 9 \mathrm{~mm}$ & 57.1 & 92.6 & 85.2 \\
$\geq 10 \mathrm{~mm}$ & 57.1 & 92.6 & 85.2 \\
\hline
\end{tabular}

longer was used as the diagnostic criterion for metastatic RLNs of head and neck cancer [18]. Recently, from the examination of RLNs in healthy subjects by MRI, a minimal axial diameter of $5 \mathrm{~mm}$ or longer was often used as the diagnostic criterion $[19,20]$. However, it is controversial to extrapolate the MRI findings of healthy individuals to the diagnostic criteria of cancer patients. Regarding RLN for MSC, there are reports of diagnostic criteria of $5 \mathrm{~mm}$ or more for the minimal axial diameter and $8 \mathrm{~mm}$ or more for the major axial diameter, and the diagnostic criteria are not constant $[14,21]$. Zhang et al. reported that the optimal diagnostic criterion was a minimal axial diameter of $6 \mathrm{~mm}$ or longer for metastatic RLNs in nasopharyngeal cancer on the basis of the treatment response by MRI before and after treatment [22]. Pathological findings in the RLN region are difficult to obtain due to the risk of adverse events such as vessel and nerve damage and dysphagia by dissection, and it is particularly useful to determine the diagnostic criteria for metastatic RLNs by imaging $\mathrm{f} / \mathrm{u}$ [23]. Therefore, we revealed the optimal diagnostic criteria for RLNs for MSC from MRI $\mathrm{f} / \mathrm{u}$.

Judgment of metastatic RLNs by MRI f/u was determined according to RECIST ver. 1.1. Notably, in the 
Table.5 Reports of recurrence of RLNs

\begin{tabular}{|c|c|c|c|c|c|c|}
\hline & Total number & $\begin{array}{l}\text { Number of RLN } \\
\text { recurrences (\%) }\end{array}$ & Stage & Pathology & Treatment & $\begin{array}{l}\text { Radiologic criteria } \\
\text { on metastatic RLN }\end{array}$ \\
\hline Kimura et al. 1998 & NR & 2 & T3N1M0, T3NOMO & SCC, ME & $S+I A R T, S+A C$ & $N R$ \\
\hline Le et al. 2000 & 97 & $2[2]$ & $\mathrm{T} 3$ or $\mathrm{T} 4$ & NR & $N R$ & $N R$ \\
\hline Umeda et al. 2005 & 22 & $1[5] ※$ & T2NOMO & SCC & $S+I A R T$ & $N R$ \\
\hline Homma et al. 2014 & 98 & $2[2]$ & T4NOMO & SCC & $S+R T$ & NR \\
\hline Jeon et al. 2017 & 71 & $1[1] \circledast$ & $N R$ & $N R$ & NR & NR \\
\hline
\end{tabular}

※contralateral side recurrence

SCC; squamous cell carcinoma, ME; mucoepidermoid cancer, S; surgery, IART; intraarterial chemotheraoy and radiation therapy

case of continuous SD, the pretreatment image and the most changed image were compared to prevent an erroneous SD judgment. As a result, there were no cases of SD to PR or PD. On the basis of the therapeutic response, seven in 34 RLNs were classified as positive for metastasis. RLNs are classified as lateral and medial, and medial RLNs are usually judged to be metastatic by visual recognition, regardless of size [24]. There was no medial RLN, and thus there was no effect on the size in the radiologic criteria. Although not statistically significant, the maximal axial diameters of metastatic positive RLNs tended to be greater than those of negative RLNs, which reflected the findings of Zhang et al. Metastatic RLNs were more common at the occipital bone level than in previous studies [20], which may be because the primary site of this study included skull base invasion in many cases. The predictive value between anatomical location was not found (data not shown). All these metastatic RLNs were present on the ipsilateral side despite being advanced cases, and this result may indicate that prophylactic irradiation of the RLN region is sufficient on the ipsilateral side alone.

From the ROC curve analysis, metastatic RLNs tended to be diagnosed more accurately with the minimal axial diameter than with the maximal axial diameter. This was consistent with previous studies of RLNs in head and neck cancer [22, 24]. The most appropriate radiologic criterion was a minimal axial diameter of $5 \mathrm{~mm}$ or more, similar to the results reported by Zhang et al. in nasopharyngeal cancer. Thus, the diagnostic criterion revealed by this study was the same as those commonly used for head and neck cancer from the examination of RLNs in healthy subjects by MRI [19, 20]. Regarding the case with false positive in this criterion, the therapeutic response was judged to be SD. However, the $\mathrm{f} / \mathrm{u}$ was performed only once, and caution is required in its interpretation. There were three RLNs with central necrosis, all of which were metastatic RLNs by MRI $\mathrm{f} / \mathrm{u}$ and the specificity was $100 \%$. The minimal axial diameter in one of three cases with central necrosis was $4.4 \mathrm{~mm}$, and the addition of this finding to the diagnostic criterion by minimal diameter increased sensitivity slightly. However, the only findings of central necrosis of a small LN with a minimal axial diameter of approximately $5 \mathrm{~mm}$ were considered to have low sensitivity. That is, it was determined that the findings of central necrosis could be replaced by the criterion of minimal diameter, because only one case of central necrosis deviated from the criterion by minimal diameter.

This study has several limitations associated with its retrospective design. First, the modality and protocol of MRI performed before and after treatment were not standardized. Second, no pathological examination was performed, and positive metastasis was only identified by MRI f/u.

In this study, no RLN recurrence was observed, and the necessity of irradiation in the RLN region remains unknown. In recent years, there have been several reports of RLN recurrence (Table 5) [25-29], and in the future, the requirement for irradiation in the RLN region should be examined by evaluating the recurrence type using our radiologic criteria, taking into consideration any adverse events.

\section{Conclusion}

In conclusion, the most appropriate radiologic criterion of metastatic RLNs in patients with MSC is a minimal axial diameter of $5 \mathrm{~mm}$ or more detected by MRI f/u.

\section{Acknowledgements}

We thank H. Nikki March, PhD, from Edanz (https://jp.edanz.com/ac) for editing a draft of this manuscript.

\section{Authors' contributions}

YK and MS analyzed and interpreted the data. YK prepared the manuscript, conducted the literature search, and reviewed and edited the manuscript. YK, MS, MF, SO, FM, YM, TK, MO, NS, and KS reviewed the manuscript. All authors have read and approved the final manuscript.

Funding

No funding was received for this study. 


\section{Availability of data and materials}

The data that support the findings of this study are available on request from the corresponding author.

\section{Code availability}

Not applicable.

\section{Declarations}

\section{Ethics approval and consent to participate}

This retrospective analysis was approved by the ethics committee of Juntendo University (approval number: 19-173). Informed consent was obtained from allindividual participants included in the study.

\section{Consent for publication}

Informed consent was obtained from all individual participants included in the study.

\section{Competing interests}

All authors declare that they have no competing interests.

\section{Author details}

'Department of Radiation Oncology, Juntendo University, 2-1-1 Hongo, Bunkyo-ku, Tokyo 113-8421, Japan. ' Department of Otorhinolaryngology, Head and Neck Surgery, Juntendo University, Tokyo, Japan. ${ }^{3}$ Department of Radiology, Juntendo University, Tokyo, Japan.

\section{Received: 18 May 2021 Accepted: 15 September 2021} Published online: 26 September 2021

\section{References}

1. Abu-Ghanem S, Horowitz G, Abergel A, Yehuda M, Gutfeld O, Carmel NN, et al. Elective neck irradiation versus observation in squamous cell carcinoma of the maxillary sinus with NO neck: A meta-analysis and review of the literature. Head Neck. 2015;37(12):1823-8.

2. Siddiqui F, Smith RV, Yom SS, Beitler JJ, Busse PM, Cooper JS, et al. ACR appropriateness criteria((R)) nasal cavity and paranasal sinus cancers. Head Neck. 2017;39(3):407-18

3. Homma A, Oridate N, Suzuki F, Taki S, Asano T, Yoshida D, et al. Superselective high-dose cisplatin infusion with concomitant radiotherapy in patients with advanced cancer of the nasal cavity and paranasal sinuses: a single institution experience. Cancer. 2009;115(20):4705-14.

4. Homma A, Sakashita T, Yoshida D, Onimaru R, Tsuchiya K, Suzuki F, et al. Superselective intra-arterial cisplatin infusion and concomitant radiotherapy for maxillary sinus cancer. Br J Cancer. 2013;109(12):2980-6.

5. Yokoyama J, Ohba S, Fujimaki M, Anzai T, Kojima M, Ikeda K, et al. Impact of intra-arterial chemotherapy including internal carotid artery for advanced paranasal sinus cancers involving the skull base. Br J Cancer. 2014;111(12):2229-34.

6. NCCN. National Comprehensive Cancer Network (2019) NCCN, Clinical Practice Guidelines in Oncology Head and Neck Cancers, version 3. https://www.nccn.org/professionals/physician_gls/pdf/head-and-neck. pdf. Accessed 21 July 2020.

7. Kosugi Y, Kawamoto T, Oshima M, Fujimaki M, Ohba S, Matsumoto F, et al. Invasion of the pterygoid plates: an indicator for regional lymph node failure in maxillary sinus cancer. Radiat Oncol. 2021;16(1):2.

8. Kosugi Y, Kawamoto T, Oshima M, Suzuki M, Fujimaki M, Ohba S, et al. Potential retropharyngeal lymph node metastasis on diagnostic image and changes in retropharyngeal regional dose depending on the irradiation technique in maxillary sinus cancer. Jpn J Head Neck Cancer. 2021;47(1):47-52.

9. Iyizoba-Ebozue Z, Murray LJ, Arunsingh M, Vaidyanathan S, Scarsbrook AF, Prestwich RJD. Incidence and patterns of retropharyngeal lymph node involvement in oropharyngeal carcinoma. Radiother Oncol. 2020;142:92-9.

10. Feng FY, Kim HM, Lyden TH, Haxer MJ, Feng M, Worden FP, et al. Intensitymodulated radiotherapy of head and neck cancer aiming to reduce dysphagia: early dose-effect relationships for the swallowing structures. Int J Radiat Oncol Biol Phys. 2007;68(5):1289-98.

11. Mazzola R, Ricchetti F, Fersino S, Fiorentino A, Giaj Levra N, Di Paola G, et al. Predictors of mucositis in oropharyngeal and oral cavity cancer in patients treated with volumetric modulated radiation treatment: A dosevolume analysis. Head Neck. 2016;38(Suppl 1):E815-9.

12. Mazzola R, Ricchetti F, Fiorentino A, Fersino S, Giaj Levra N, Naccarato S, et al. Dose-volume-related dysphagia after constrictor muscles definition in head and neck cancer intensity-modulated radiation treatment. $\mathrm{Br} J$ Radiol. 2014;87(1044):20140543.

13. Mendenhall WM, Mancuso AA. Radiotherapy for head and neck cancer-is the "next level" down? Int J Radiat Oncol Biol Phys. 2009;73(3):645-6.

14. Guan X, Wang X, Liu Y, Hu C, Zhu G. Lymph node metastasis in sinonasal squamous cell carcinoma treated with IMRT/3D-CRT. Oral Oncol. 2013;49(1):60-5.

15. Biau J, Lapeyre M, Troussier I, Budach W, Giralt J, Grau C, et al. Selection of lymph node target volumes for definitive head and neck radiation therapy: a 2019 update. Radiother Oncol. 2019;134:1-9.

16. LH s. TNM Classification of Malignant Tumours, UICC, 7th edn. New York: Wiley-Liss. 2009.

17. Eisenhauer EA, Therasse P, Bogaerts J, Schwartz LH, Sargent D, Ford R, et al. New response evaluation criteria in solid tumours: revised RECIST guideline (version 1.1). Eur J Cancer. 2009;45(2):228-47.

18. Mancuso AA, Harnsberger HR, Muraki AS, Stevens MH. Computed tomography of cervical and retropharyngeal lymph nodes: normal anatomy, variants of normal, and applications in staging head and neck cancer. Part II: Pathol Radiol. 1983;148(3):715-23.

19. King AD, Ahuja AT, Leung SF, Lam WW, Teo P, Chan YL, et al. Neck node metastases from nasopharyngeal carcinoma: MR imaging of patterns of disease. Head Neck. 2000;22(3):275-81.

20. Liu LZ, Zhang GY, Xie CM, Liu XW, Cui CY, Li L. Magnetic resonance imaging of retropharyngeal lymph node metastasis in nasopharyngeal carcinoma: patterns of spread. Int J Radiat Oncol Biol Phys. 2006;66(3):721-30.

21. Watarai J, Seino Y, Kobayashi M, Shindo M, Kato T. CT of retropharyngeal lymph node metastasis from maxillary carcinoma. Acta Radiol. 1993;34(5):492-5

22. Zhang GY, Liu LZ, Wei WH, Deng YM, Li YZ, Liu XW. Radiologic criteria of retropharyngeal lymph node metastasis in nasopharyngeal carcinoma treated with radiation therapy. Radiology. 2010;255(2):605-12.

23. Shellenberger T, Fornage B, Ginsberg L, Clayman GL. Transoral resection of thyroid cancer metastasis to lateral retropharyngeal nodes. Head Neck. 2007:29(3):258-66

24. Coskun HH, Ferlito A, Medina JE, Robbins KT, Rodrigo JP, Strojan P, et al. Retropharyngeal lymph node metastases in head and neck malignancies. Head Neck. 2011;33(10):1520-9.

25. Kimura Y, Hanazawa T, Sano T, Okano T. Lateral retropharyngeal node metastasis from carcinoma of the upper gingiva and maxillary sinus. AJNR Am J Neuroradiol. 1998;19(7):1221-4.

26. Le QT, Fu KK, Kaplan MJ, Terris DJ, Fee WE, Goffinet DR. Lymph node metastasis in maxillary sinus carcinoma. Int J Radiat Oncol Biol Phys. 2000;46(3):541-9.

27. Umeda M, Minamikawa T, Komatsubara H, Ojima Y, Shibuya Y, Yokoo S, et al. En bloc resection of the primary tumour and cervical lymph nodes through the parapharyngeal space in patients with squamous cell carcinoma of the maxilla: a preliminary study. Br J Oral Maxillofac Surg. 2005:43(1):17-22

28. Homma A, Hayashi R, Matsuura K, Kato K, Kawabata K, Monden N, et al. Lymph node metastasis in 44 maxillary sinus squamous cell carcinoma: incidence and treatment outcome. Ann Surg Oncol. 2014;21(5):1706-10.

29. Jeon $\mathrm{SH}, \mathrm{Han} \mathrm{DH}$, Won TB, Keam B, Kim JH, Wu HG. Implication of tumor location for lymph node metastasis in maxillary sinus carcinoma: indications for elective neck treatment. J Oral Maxillofac Surg. 2017;75(4):858-66.

\section{Publisher's Note}

Springer Nature remains neutral with regard to jurisdictional claims in published maps and institutional affiliations. 